\title{
Politische Strukturen eines Dorfes in Bangladesh*
}

\author{
von Dietmar Herbon
}

\section{Einleitung}

Bangladesh ist eines der ärmsten und am dichtesten bevölkerten Länder der Erde und gilt als "Testfall" für den Erfolg von Entwicklungspolitik und verschiedener entwicklungspolitischer Strategien. Nicht alle Probleme Bangladeshs sind alleine auf die Tatsache zurückzuführen, daß die Bevölkerung schneller wächst als die landwirtschaftliche oder die industrielle Produktion. Einige sind sicherlich auch auf die Wirkungen kolonialer und internationaler Politik und auf die Vertretung der Eigeninteressen nationaler Führungsschichten zurückzuführen. ${ }^{1}$

Will man die gegenwärtigen Probleme lösen, muß man dies auf der Basis der entstandenen Strukturen tun. Seit der Unabhängigkeit Bangladeshs (1971) hat eine grundsätzliche Umorientierung von einer staatskapitalistisch orientierten Industrialisierungspolitik zu einer eher privatwirtschaftlich orientierten ländlichen Entwicklungspolitik stattgefunden. ${ }^{2}$ Die von den letzten bangladeshi Regierungen deklarierte Entwicklungsstrategie ${ }^{3}$ basiert auf der Förderung ländlicher und landwirtschaftlicher Entwicklung mit entsprechenden komplementären Maßnahmen und mit dem Ziel, die landwirtschaftliche Produktion soweit zu steigern, daß sie mit dem Bevölkerungswachstum Schritt hält. Im Rahmen der Diskussionen von Strategien zur ländlichen Entwicklung werden u. a. auch Uberlegungen im Hinblick auf eine adäquate Partizipation der betroffenen Bevölkerung oder der entsprechenden Zielgruppen angestellt. Die Berücksichtigung und Einbeziehung der Bedürfnisse der Betroffenen bei Zieldefinition, Projektfindung, -planung, -durchführung und -bewertung setzt eine gründliche Kenntnis sozio-politischer Strukturen und Prozesse an den "Graswurzeln" voraus. ${ }^{4}$

* Ergebnisse einer empirischen Studie.

1 Siehe als kurze Einführungen in die Problematik Bangladeshs u. a.: Nohlen, D., Lexikon der Dritten Welt, Baden-Baden, 1980, S. 42 ff; Zingel, W.-P., Bangladesh, in: Nohlen, D., Nuscheler, F. (Hrsg.) Handbuch der Dritten Welt, Band 4., Unterentwicklung und Entwicklung in Asien, Halbband I., Afghanistan - Laos, Hamburg 1978, S. 39 ff; de Vylder, S., Asplund, D., Contradictions and Distortions in a Rural Economy The Case of Bangladesh; Report from Policy Development and Evaluation Division, Sida 1977.

2 Für eine kurze Beschreibung siehe: Herbon, D., Ein dörfliches Gesellschaftssystem in Bangladesh Mechanismen, Funktionen und Dynamik des sozio-ökonomischen Austausches, Göttingen 1984 S. 8 ff.

3 Vgl. The Second Five Year Plan 1980-85, Draft, Planning Commission, Government of the People's Republic of Bangladesh, Dacca 1980.

4 Siehe z. B. Müller, J. O., Soziale Partizipation - Konzept, Probleme und Bedingungen eines entwicklungspolitischen Ideals, S. 57-67, in: Rurale Entwicklung zur Uberwindung von Massenarmut, Hrsg. von Groeneveld, S. und Meliczek, H., Saarbrücken 1978; Herbon, D., Zur Bedeutung des 
Diese Kenntnis ist sehr wichtig für eine realistische und erfolgreiche Entwicklungspolitik, denn es hat den Anschein, daß ihre Nichtberücksichtigung oft schwerwiegende Folgeprobleme bedingt und zuweilen wohl auch zum Scheitern der gesamten Strategie führt. Andererseits scheint es auch auf der Seite der "Entwicklungsplaner" so zu sein, daß soziale und politische Strukturen von Entwicklungsgesellschaften als Phänomene der "Makroebene" gesehen werden. Politische Strukturen sind jedoch nicht alleine auf eine "nationale" Ebene beschränkt, sondern sie sind durchgängig auf allen Ebenen zu finden, bis hin zur dörflichen "Mikropolitik". Dörflich-ländliche Strukturen sind nicht nur Resultat kolonialer oder nationaler Politik und internationaler Interessen, sondern besitzen durchaus eine soziale und kulturelle Eigendynamik, die zwar nicht im Einzelfall, wohl aber in der Summe, auch Rückwirkungen auf nationale Politiken haben kann. Nationale Entwicklungsplanung, bei der oft genug die Partizipation der Bevölkerung im wesentlichen als Mittel zur effizienteren Umsetzung top-down entwickelter Modelle verstanden wird, ${ }^{5}$ muß bei Nichtberücksichtigung gegebener Strukturen an der Basis mit einem Scheitern rechnen, sei es weil sie Potentiale nicht erkennen oder weil sie Widerstände nicht umgehen kann. Zudem gehen entwicklungsstrategische Vorstellungen oft mehr oder weniger bewußt entweder von homogenen Lebens- oder Interessenlagen ländlicher Bevölkerung aus, oder sie vermeinen duale Klassenstrukturen zu erkennen, denen durch entsprechend radikale Strategien beizukommen sei.

Sozio-ökonomische und politische Strukturen sind jedoch nicht auf einfache Grundbestandteile zu reduzieren. Vielmehr handelt es sich auch bei lokalen sozial-ökonomischen und politischen Strukturen um komplexe Systeme, deren Kenntnis und Berücksichtigung erst eine verantwortungsvolle Politik ermöglicht. Die Kenntnis mikropolitischer Strukturen ist eine der Voraussetzungen für ihre Nutzung oder Beeinflussung im Interesse großer Bevölkerungsteile, denen ja eine größere Chance zur Interessenartikulation, -wahrnehmung und eventuell -durchsetzung gegeben werden soll, deren aktive Partizipation also gefördert werden soll.

Andererseits ist die Kenntnis der lokalen Strukturen und Prozesse wiederum Voraussetzung für realistische Pläne. So hat es keinen Zweck von idealistischen, z. B. "demokratischen", Dorfvorstellungen auszugehen und damit letzten Endes doch wieder nur in die Hände lokaler Potentaten zu arbeiten. Vielmehr muß man sich darüber klar sein, daß mit dem Austauschen oder Abschaffen nationaler Eliten, mikropolitische Strukturen und Probleme noch lange nicht gelöst und Fragen der Partizipation noch lange nicht beantwortet sind.

In diesem Zusammenhang soll nun etwas eingehender auf "mikro-" oder dorfpolitische Strukturen eingegangen werden. Die sozio-politischen Strukturen und Prozesse in ländlichen Gesellschaften sind komplex. "It is . . . a field of tension, full of intelligent and determined antagonists, sole and corporate, who are motivated by ambition,

sozioökonomischen Austauschsystems in Dorfgemeinschaften im Hinblick auf eine integrierte ländliche Entwicklung, Saarbrücken u. a. 1981, S. $114 \mathrm{ff}$.

5 Vgl. Goetze, D.; Entwicklungssoziologie, München 1976, S. 147 ff. 
altruism, self-interest, and by desire for the public good, and who in successive situations are bound to one another through self-interest, or idealism - and separated or opposed through the same motives. At every point in this process we have to consider each unit in terms of its independent objectives, and we also have to consider the entire situation in which their interdependent actions occur." ${ }^{6}$

Auf der Grundlage der Ergebnisse einer Dorfuntersuchung in Bangladesh ${ }^{7}$ sollen hier die sozio-politische Führungsstruktur und der politische Prozeß dargestellt werden. Den folgenden Ausführungen liegt dabei folgende Definition zugrunde: "The study of politics . . . is the study of the process involved in determining and implementing public goals and in the differential achievement and use of power by the members of the group concerned with these goals. ${ }^{8}$

\section{Positionen, Kriterien und Ebenen der sozio-politischen Führungsstruktur}

Soziale Struktur zeigt sich in der Ordnung von Menschen und davon abgeleitet der Haushalte/Familien zueinander, d. h. in der Unter- und Uberordnung von Haushalten. ${ }^{9}$ Haushalte und Haushaltsvorstände, deren Wort Gewicht hat, die Einfluß und eine gewisse Macht über andere Haushalte haben, werden hier als Führer oder Führerhaushalte bezeichnet. Führer, Führerschaft und Führung sind im traditionellen System nichtformale Institutionen. Durch ihre Integration in das moderne System werden teilweise neue formale Führungsstrukturen geschaffen und traditionelle Institutionen formalisiert.

Führerschaft ist eine vertikale Beziehung zwischen Haushaltsvorständen, die im Zusammemhang mit anderen horizontalen Beziehungen zu sehen ist. Sie ist auf der Ebene des Dorfes zwar ein wirtschaftliches, politisches und kulturelles Phänomen, soll hier jedoch als sozio-politische Institution verstanden werden.

Führerschaft als soziales Phänomen erklärt sich nicht nur aus dem Bedürfnis und der Notwendigkeit der sozialen Organisation, sondern auch der wirtschaftlichen Lage der Haushalte. Sie wird kulturell bestimmt. Sie begründet sich auf verschiedenen sozialen

6 Swartz, M.J., Turner, V. W., Tuden, A., Political Anthropology, New York 1966, S. 8.

7 Siehe Herbon, D., 1984 a.a.O. Hier sei auch auf andere, für den allgemeinen Themenkreis "Mikropolitik" interessante Studien verwiesen: Islam, A. K. M. A.; A Bangladesh Village - Conflict and Cohesion, An Anthropological Study of Politics, Cambridge, Mass. 1974; Chowdhury, A., Agrarian Social Relations and Rural Development in Bangladesh, Totowa N. J. 1982, S. 51 ff. Blok, A., Coalitions in Sicilian Peasant Society, S. 151-165, Jongmans, D. G., Politics on the Village Level, S. 167-217 und Thoden van Velzen, H. U.E., Coalitions and Network Analysis, S. 219-250 in: Boissevain, J./Mitchell, J. C. (eds.), Network Analysis - Studies in Human Interaction, The Hague, Paris 1973, Scott, J. C., The Erosion of Patron-Client Bonds and Social Change in Rural Southeast Asia, in: The Journal of Asian Studies Vol. 32, No. 11972 S. 5-38. Scott, J. C., The Moral Economy of Peasants - Rebellion and Subsistence in Southeast Asia, New Haven etc. 2nd print 1977.

8 Swartz, M.J., u. a. 1966 a.a.O., S. 7.

9 Bei der Untersuchung der Gesellschaftsstruktur des bangladeshi Dorfes wurde der Haushalt als analytische Basiseinheit definiert. Die Vorstände der Haushalte wurden als die relevanten Entscheidungsträger - eine Abstraktion - aufgefaßt. Diese Definition und Setzung soll auch hier gelten. 
Ebenen unterschiedlich. Auch die.Bedeutungen und Wirkungen ändern sich. Deshalb soll hier unterschieden werden zwischen Führerschaft auf der Ebene der Baris (Gehöfte), der Paras (Weiler), des Dorfes und dann der außerdörflichen Ebene. Führer können außerdem die sogenannten "Meinungsführer" und "change agents" sein. Von ihnen gibt es grundsätzlich zwei Typen, den traditionell orientierten Mullah und den modernen relativ gebildeten Lehrer.

Eine besondere Gruppe sind die Arbeiterführer, die sowohl für die Arbeitgeber als auch für die Arbeiter eine Vermittler- und Führungsrolle spielen. Sie sind der übrigen Machthierarchie unter- oder eingeordnet.

In den folgenden Abschnitten soll der Versuch gemacht werden, diese sechs im Dorf empirisch erfaßten Führungspositionen und -ebenen darzustellen und die Kriterien, die den jeweiligen Positionen zugrunde liegen, herauszuarbeiten. Führungspositionen erfordern jeweils verschiedene Qualifikationen ihrer Inhaber, wie wirtschaftlichen Erfolg und wirtschaftliche Leistungsfähigkeit, zugeschriebene soziale Rolle und erworbenes Ansehen, Alter, Geschlecht und Verfügung über Informationen. Die Gewichtung und Bedeutung verschiebt sich jedoch von einer Führungsebene zur anderen.

\subsection{Bariführer}

Im Untersuchungsdorf gibt es ca. 100 Baris mit durchschnittlich dreieinhalb Haushalten pro Bari und zwei Personen, die als Bariführer bezeichnet werden. In kleineren Baris gibt es nur einen, in größeren mehrere Führer.

Die Rollendefinition eines Bariführers (barir malik) ist recht ungenau, weil es sich nur um eine unbedeutende und informale Position handelt. Bariführer von Baris, die nur einen Haushalt haben, qualifizieren sich einfach, weil sie der Haushaltsvorstand und Ernährer der Familie sind und als Mann automatisch Autorität über Frauen haben. Bei einem Zwei-Haushalts-Bari ist aber mindestens noch das Merkmal des Alters von Bedeutung. Es ist dann in der Regel der ültere von zwei Brüdern, der aufgrund seines Alters und der Erstgeburt vom jüngeren Bruder respektiert wird - werden muß - und insofern durch die ihm so verliehene Autorität zum Bariführer wird. Dieses Kriterium gilt auch für größere Baris. Es kommen aber, und dieses Phänomen wird mit wachsender Barigröße bedeutender, noch persönliche Qualifikationsmerkmale hinzu. Solche persönlichen Qualifikationsmerkmale sind etwa Intelligenz, individueller wirtschaftlicher Erfolg, und die Fähigkeit, sich Gehör zu verschaffen, aber auch die Einschätzung durch andere, für die er ein Vorbild in seinen Handlungen und seinem Verhalten ist. Daneben gibt es aber auch noch das Qualifikationsmerkmal der "Muskelkraft". Einige Führer zeichnen sich dadurch aus, daß sie auch mal persönlich zum Hilfsmittel der physischen Gewalt greifen. Sie erwerben sich auf diese Weise eine gewisse Art von Furcht-Respekt. Selbstverständlich ist dies nicht ihr einziges Qualifikationsmerkmal, denn physische Gewalt alleine würde schnell zu unlösbaren Konflikten, eventuell zur physischen Liquidierung führen. Es spielt daher für die Ebene der Baris insgesamt eine untergeordnete Rolle. 
Obwohl Bariführer im allgemeinen Männer sind, gibt es doch Fälle, in denen Frauen Witwen - Haushaltsvorstände und damit auch mehr oder weniger bedeutende Bariführer sind. Dies ist der Fall bei Witwen, die nicht zu ihrer Familie zurückkehren wollen oder können und deren Söhne noch nicht alt genug sind, selbst Ansehen zu genießen. Es kann aber auch sein, daß es sich um alte aus wohlhabenden Familien stammende Witwen handelt, die kraft Persönlichkeit die Fäden, auch über ihre Söhne, noch in der Hand halten.

Neben den oben geschilderten Kriterien spielen selbstverständlich auch wirtschaftliche Kriterien eine Rolle. Bariführer unterscheiden sich von Nicht-Bariführern durch eine größere Kontrolle über Land (Eigentum, Besitz), und zwar umso mehr, je mehr sie als Bariführer angesehen werden. Es existiert ein Zusammenhang zwischen Ressourcenkontrolle, eventuell auch Erfolg, und Bariführerschaft. Dieser Zusammenhang zeigt sich beim Haushalts- und Pro-Kopf-Einkommen jedoch nicht. Es muß daher angenommen werden, daß Bariführer nicht respektiert werden, weil sie reich an sich sind, vielmehr, daß es wohl ältere Haushaltsvorstände sind, die noch die Ressourcen kontrollieren, während z. B. Söhne zwar eigene Haushalte haben, aber auf ihre Väter angewiesen sind. Die Bariführungsstruktur hängt eng zusammen mit der Struktur des Baris selbst. Die Baris der Landbewirtschafter sind im Schnitt größer. Landbewirtschaftende Haushalte und Baris sind im Prinzip hierarchisch strukturiert und auf einen Patron hin orientiert, während z. B. Arbeiterhaushalte isolierter und individualisierter sind und auch die Führer dieser Haushalte und Baris mehr auf ihre Unabhängigkeit und Eigenständigkeit bedacht sind. Im allgemeinen dominieren die großen, herausragenden Baris mit ihrem zentralen Haushalt, auch auf der nächsten Ebene, dem Para oder Parateil.

\subsection{Paraführer}

Baris vereinigen sich zu größeren Agglomerationen. Wie einzelne Haushalte sich in Baris organisieren und in eine Führungsstruktur eingliedern, geschieht gleiches mit Haushalten und Baris auf Paraebene.

Paraführer haben gegenüber Bariführern ein leicht verschobenes Qualifikationsprofil. In der Regel sind Paraführer auch gleichzeitig Führer eines eigenen Baris. Es gibt aber auch Ausnahmen. In den Baris ist das Kriterium des Alters und der verwandtschaftlichen Unter- und Uberordnung eindeutig dominierend. Das ist es auf Paraebene für einen Paraführer nicht bzw. nicht zwangsläufig. So kann es sein, daß sich ein relativ junger Mann im Bari seinem Vater oder älteren Bruder unterordnet, sei es, weil es sich so gehört, sei es, weil der andere die Ressourcen kontrolliert, während er auf Paraebene als eine zentrale Persönlichkeit anerkannt wird, deren Wort Gewicht hat.

Damit ist schon gesagt, daß ein wichtiges Kriterium für die Führerschaft auf Paraebene die persönliche Eignung und Fähigkeit ist. Hinzu kommen als Kriterien sicher auch das Alter und die "Familie", aus der er kommt. Alter für sich genommen reicht nicht aus, obwohl alten Männern relativ viel Respekt gezollt wird. Tatsächlich sind aber die alten Männer der landbesitzenden Verwandtschaftsgruppen, wenn Führungsanspruch und 
Führungsfähigkeit hinzukommen, potentielle Para-Führungspersönlichkeiten. Aber auch ein aufgrund seiner Persönlichkeit allgemein akzeptierter und beliebter Arbeiter kann für eine Anzahl ärmerer Arbeiterhaushalte zu einer legitimen Führungspersönlichkeit werden, jedenfalls im nachbarschaftlichem Umkreis, etwa einem Teil des Paras. In einem Para gibt es immer mehrere Führer, die sich aufgrund unterschiedlicher Merkmale qualifizieren und die u. U. um Einfluß konkurrieren. Die bedeutenden Paraführer sind in der Regel wirtschaftlich dynamische und erfolgreiche Menschen, was sich z. B. darin zeigt, daß sie, was Landeigentum angeht, zu den Mittel- und Kleineigentümern gehören. Es gelingt ihnen offensichtlich, durch Teilpacht und Geldpacht die ihnen zur Verfügung stehende Landfläche zu vergrößern. Damit vergrößert sich auch das Einkommen des Haushalts. Somit korreliert die erfolgreiche Kontrolle und Nutzung von Ressourcen eng mit dem Erwerb von Ansehen im Dorf und bedingt neben den persönlichen Führungseigenschaften die Qualifikation und Anerkennung als Paraführer.

Kontrolle von Ressourcen ist allerdings nur dann von Bedeutung, wenn sie auch anderen, nämlich der Klientel nützt. Paraführer haben überdurchschnittlich viele Verwandte im Dorf. Eine gewisse Rolle bei der Durchsetzung als Führer spielt die Verwandten-Klientel, die ihren Mann stützt, ein Prinzip, das auch für Führer auf Dorfebene gilt.

\subsection{Führer auf Dorfebene}

Als Führer auf Dorfebene ${ }^{10}$ sollen die Führer verstanden werden, die von Parabewohnern aus allen Paras als Führungspersönlichkeiten des ganzen Dorfes angesehen und bezeichnet wurden. Das heißt nicht, daß ein Dorfführer von allen Haushalten anerkannt sein muß, sondern nur von sehr vielen. Dorfführer sind im Grunde nichts weiter als hervorragende Paraführer. Das Qualifikationsmerkmal der Persönlichkeit tritt hinter dem der Ressourcenkontrolle, d. h. besonders des herausragenden Landeigentums und auch Einkommens, zurück. Dorfführer sind in der Regel auch schon recht alte Männer, in gewisser Weise die Patriarchen des Dorfes, die aufgrund ihres Alters und of auch wegen ihrer Weisheit, Klugheit und politischen Erfahrung einen weit überdurchschnittlichen Respekt genießen. Eine zweite Kategorie von Dorfführern sind etwas jüngere Männer, die durch ihr Auftreten als Paraführer über ihr Para hinaus bekannt und anerkannt sind. Auch sie gehören zu den landbesitzenden Schichten.

Diese Dorfführer, die sich also durch herausragende Ressourcenausstattung und durch altersbedingte Erfahrung qualifizieren, sind oft schon so alt, daß sie sich nicht mehr im sozio-politischen Tagesgeschäft engagieren und - als Paraführer - dieses Geschäft den jüngeren und dynamischeren Männern überlassen, oft ihren schon "mittelalterlichen" Söhnen. Nur bei außergewöhnlichen Entscheidungen, die ihr Para und das Dorf als

Der Einfachheit halber soll von hier ab von Dorfführern gesprochen werden. 
Ganzes betreffen, werden sie aktiv und tun ihre Meinung kund. Ohne und gegen sie geht dann im Ernstfall nichts.

Es gibt noch eine zweite Gruppe von Dorfführern, das ist in gewisser Weise der Nachwuchs. Diese jungen Männer sind oft Paraführer, sind in der täglichen Dorfpolitik engagiert und müssen über ähnliche Qualifikationen verfügen wie alle Führungspersönlichkeiten, nämlich über weit überdurchschnittlich viel Land und Einkommen. Sie müssen über recht viele Beziehungen verfügen und im allgemeinen mindestens Lesen und Schreiben können. Sie sind also die Führer "im Wartestand", die viele Dinge selbständig regeln, aber, solange der alte Patriarch noch lebt, nicht das letzte Entscheidungsrecht haben.

Dorfführer rekrutieren sich aus den Landeigentümerhaushalten. Es handelt sich also um die traditionelle agrarische Führungsschicht. Arbeiter und Händler können bei entsprechendem Engagement und entsprechenden Fähigkeiten wohl als Paraführer anerkannt werden, als traditionelle und einflußreiche Repräsentanten des Dorfes werden sie kaum auftreten.

\subsection{Führer im kognitiven Bereich}

Neben den bisher genannten Führern gibt es noch andere Führungspersönlichkeiten und -strukturen, die nicht direkt in dieses hierarchisch-vertikale System integriert sind. Diese parallelen und/oder alternativen Führer können einerseits die modernen "change agents" sein, die sich durch die Kontrolle von Informationen der außer-ruralen Welt qualifizieren. Andererseits gibt es traditionelle sozio-religiöse Autoritäten, Imame und Moulana, die kraft traditionellen und religiösen Wissens, Wertevermittlung und -kontrolle Führungsfunktionen im normativen Bereich innehaben. Diese modernen oder traditionellen Informations- und Wertevermittler sind idealtypisch zu unterscheiden von außenorientierten Führern, die of $t$ nicht im dörflichen System begründete Führungsansprüche haben, sondern bis zu einem gewissen Grade außerdörfliche Ressourcen kontrollieren, also für sich und/oder ihre Klientel Ressourcen z. B. des Staates anzapfen, umleiten und verteilen können.

Bei diesen zwei Führertypen handelt es sich in gewisser Weise um Führungsspezialisten, die allerdings darauf aus sind, ihre Basis, ihr Ansehen und ihren Status im Dorf zu vergrößern.

Imame (traditionelle religiöse Führer) sind im Prinzip Vorsteher der religiösen Gemeinschaften (jamat), die durch Koranlesungen und -interpretation den Dorfbewohnern die Werte und Normen des Islam zu vermitteln suchen. Diese Führer greifen mit mehr oder weniger großem Erfolg stark in den kognitiven Bereich der Dorfgemeinschaft ein. Ihr Erfolg zeigt sich nicht so sehr im materiellen Bereich als vielmehr im sozialen. Imame sind in gewisser Weise Laien, die, wenn sie selbst kein Land bewirtschaften, auf den Unterhalt durch ihre Gemeinde angewiesen sind und daher keine bedeutenden materiellen Werte akkumulieren können. Sie haben eine Führungs- und Spezialisten- 
funktion im kognitiven Bereich und sind getrennt zu sehen von sonstigen sozio-politischen Führern.

Neben den traditionellen "Meinungsmachern", den Imamen, gibt es noch Persönlichkeiten (change agents), die zwischen "modernen" - "externen" - kognitiven Bereichen und dem "traditionellen" - "internen" - System vermitteln und ein recht hohes Ansehen genießen. Es sind im Fall des Untersuchungsdorfes hauptsächlich die Lehrer. Lehrer gehören in der Regel zu den Wohlhabenden im Dorf. Ihr Einkommen ermöglicht ihnen einen recht hohen Lebensstandard und Investitionen in Land. Sie genießen daher schon aufgrund ihrer materiellen Lage ein relativ hohes Ansehen, das sich alleine aufgrund der Tatsache, daß sie etwas gelernt haben (shikito), noch vergrößert. Sie gehören nicht zu den traditionellen Führern, haben jedoch aufgrund der Tatsache, daß "studierten" Leuten Respekt gezollt wird, im Rahmen einer "sozialen Aufmerksamkeitsstruktur" einen gewissen "modernen", "aufgeklärten" Einfluß.

Die Lehrer demonstrieren, daß man durch harte Arbeit auch wirtschaftlich erfolgreich sein kann. Sie sind oft entweder als nebenberufliche Landbewirtschafter oder Händler aktiv und erfolgreich. Charakteristischerweise sind die Lehrer nicht so ganz in das dörfliche Macht- und Sozialsystem integriert. Sie sind ebenso wie Imame kein realer Machtfaktor, keine Patrone mit Klientel. Aber sie sind hoch respektiert und als Gruppe, die das kognitive System nicht nur der Schüler, sondern auch ihrer dörflichen Nachbarn beeinflußt, sicher bedeutend.

\subsection{Außenorientierte Führer}

Es lassen sich drei Typen von Führern identifizieren, deren Aktions- und Wirkungsbereich z. T. oder ganz außerhalb des Dorfes liegt. Der erste Typ ist der des "Bazar-Politikers". Im Dorf und auch außerhalb des Dorfes im Bazar gibt es eine Reihe von einflußreichen, ja mächtigen Männern, die ihre Macht und Position nicht primär aus traditioneller Macht, Kontrolle über agrarische Ressourcen usw. ableiten, sondern aus der Kontrolle externer Ressourcen und z. T. aus der Vermittlung und Vergabe eines Teils dieser Ressourcen an eine dörfliche Klientel.

Diese Männer können z. T. reichere Dorfbewohner, Landbewirtschafter sein, die als Bari- und Paraführer über eine "Basis im Dorf verfügen. In der Regel gelingt es ihnen, über Handelstätigkeit im Bazar dort entsprechende Kontakte zu knüpfen. Auf diese Weise können sie einen relativ großen Reichtum erwerben, der dann zu einem Teil ins Dorf zurückfließt. Dies kann u. U. zu einer Steigerung der Popularität, aber auch einfach zu einer Machtbasis führen, die sich aus der Kontrolle dörflicher oder externer Ressourcen ergibt. Außerdem sind sie aber auch in der "Bazar-Politik" aktiv und wirken so z. T. in einem überlokalen Rahmen.

Der zweite Typ dieser modernen Führer gründet seine Macht auf seine Position in Parteiapparaten oder staatlichen Institutionen. Diese Position resultiert z. T. aus gewissen Rollen und Verbindungen aus der Zeit des Unabhängigkeitskampfes gegen 
Pakistan und der Staatsgründungsphase. Dies ist einer der Gründe dafür, warum z. T. recht junge Leute sehr einflußreiche Positionen im Bazar und im Dorf innehaben. Charakteristisch für diese jungen Führer ist die Tatsache, daß sie externe Ressourcen kontrollieren, sie sich aneignen oder ihren Fluß zugunsten der Dorfbevölkerung beeinflussen können, was ihnen ihre Klientel schafft. Auf diese Weise können sie auch gute Beziehungen zu lokalen Führern pflegen. Diese Ressourcen sind z. B. staatlich kontrollierte und subventionierte Konsumgüter (Rationen) oder Produktionsgüter (landwirtschaftliche Betriebsmittel wie Pestizide, Saatgut, Wasserpumpen), aber auch Kredite oder Informationen. Zusätzlicher Einfluß erwächst ihnen aus Beziehungen zu Beamten. So gelingt es ihnen z. T. durch Bestechung, vom Staat zur Verfügung gestellte Projektmittel in ihr Dorf zu lenken (z. B. Weizen für Straßenbauprojekte im Rahmen von Food-For-Work-Programmen).

Ein dritter $T y p$ basiert auf den Beziehungen, die ein Führer unterhalten kann, weil er z. B. als Bauunternehmer im Rahmen staatlicher Programme eine Arbeiterklientel mit Arbeit und damit mit Einkommen versorgen kann. Er macht damit Abhängigen Ressourcen zugänglich, die er natürlich auch für sich selbst nutzt. Auf diese Art kann er, unter der Voraussetzung, daß er zuerst über ein gewisses Eigenkapital verfügt, sich selbst einen hohen Lebensstandard und eine eigene Klientel finanzieren, was entsprechenden Prestigegewinn bringt.

In diese Lage der Nutzung externer Ressourcen kommen oder kamen diese Führer in der Regel durch die Ubernahme von Funktionen in der jeweils herrschenden Partei. So konnten sie entweder illegal Staatsgelder abzweigen oder durch Rationenhandels- oder Bauunternehmerlizenzen und -aufträge die Kontrolle über staatliche Ressourcenflüsse im lokalen Bereich erlangen. Z. T. setzten sie sich mit Waffengewalt durch. Die drei Führertypen sind nur analytisch zu trennen, tatsächlich engagieren sich alle Führer in möglichst vielen Bereichen.

\subsection{Arbeiterführer}

Eine Position besonderer Art haben die Arbeiter- und Arbeitergruppenführer. Sie sind Vorarbeiter, Arbeitervermittler und Interessenvertreter in einem. Arbeitgeber, die eine größere Zahl von Arbeitern abwerben wollen, setzen sich mit einem Arbeiterführer in Verbindung, der dann eine Gruppe von entsprechender Größe zusammenstellt. Er ist sowohl für den Arbeitgeber als auch für die Arbeiter der Ansprechpartner. Er handelt die Löhne aus und wird in der Regel besser bezahlt als seine Arbeiter und stellt für sie eine Art Patron dar. Man ist darauf angewiesen, sich mit ihm gut zu stellen.

Arbeitergruppenführer treten nicht immer in Erscheinung, sondern nur bei bestimmten Arbeiten. Erdarbeiten und Herstellung von Ziegeln machen etwa $85 \%$ der Aktivitäten von Arbeitsgruppen aus. Landwirtschaftliche Arbeiten spielen keine Rolle. Es gibt noch einige spezializierte Gruppen, die Schreiner und Lastenträger, die sich auch in kleineren Gruppen mit Führern organisieren, aber aufs Ganze gesehen eine untergeordnete Rolle spielen. 
Arbeiterführer sind keine gewöhnlichen Arbeiter. Sie sind einmal von der Persönlichkeit her dominante und dynamische Menschen, zum anderen sind sie intelligent und auch überdurchschnittlich kräftig. Aber hinzu kommt noch, daß sie keine reinen Arbeiter sind, sondern oft noch etwas Land bewirtschaften. Außerdem gehören sie, was die Höhe ihres Haushaltseinkommens angeht, nicht zu den Ärmsten im Dorf. Diese "Zwischenklassenposition" hat für alle Vorteile. Die Arbeiter haben einen Vertreter, der sich relativ aggressiv gegen Arbeitgeber behaupten kann, denn er hat ein relativ sicheres Auftreten und kein unterwürfiges Verhalten. Die Arbeitgeber haben den Vorteil, es im Prinzip mit einem Mann zu tun zu haben, den sie eventuell leichter kontrollieren können, weil sie ihn z. T. auch als sozial nahestehend akzeptieren können, oder der im Zweifelsfall wegen seiner vereinzelten und herausgehobenen Position durch besondere materielle Zuwendungen gewonnen werden kann. Man kann ihn auch durch die Androhung des Boykotts seiner Vorarbeit und damit der ihm und seiner Klientel drohenden Arbeitslosigkeit unter Druck setzen.

\section{Führerhierarchie und Gefolgschaftsbildung}

\subsection{Führerqualifikationen auf verschiedenen Ebenen}

Die Führungsstruktur im Dorf ist recht komplex. Die unterste Ebene ist die der Bariführung. In ihr dominieren die Prinzipien der persönlichen Dominanz, des höheren Alters und zu einem gewissen Teil auch der persönlichen Fähigkeiten. Der Haushaltsvorstand, der sich im Rahmen eines Baris keinem anderen unterordnen will, kann sich trennen und damit sein eigener Bariführer werden. Bariführung kann noch zu einem gewissen Grad selbstzugeschrieben werden und ist an sich noch nicht abhängig von Ressourcenausstattung und Einkommen.

Dies ändert sich auf der nächst höheren Ebene, der Paraführung oder der Parasegmentführung. Hier spielt die persönliche soziopolitische Durchsetzungsfähigkeit und der individuelle persönliche Erfolg eine zunehmende Rolle. Alter ist nur noch von sekundärer Bedeutung. Allerdings spielt hier auch die Position des Vaters eine gewisse Rolle. Der Status als anerkannter überdurchschnittlicher Mann, eben als Paraführer, wird teilweise zugeschrieben. Hierzu kommt beim Paraführer, und hier ist der Ubergang zum Dorfführer fließend, noch die Ressourcenkontrolle und die Verfügung über überdurchschnittlich viel Einkommen.

Bei Dorfführern, die in der Regel auch Bari- und Paraführer sind, kommt allerdings noch das Kriterium der Respektabilität und des Alters hinzu.

Sowohl im Falle des Paraführers als auch in dem des Dorfführers ist der Status nicht selbst zugeschrieben, sondern er wird von einer Gefolgschaft zugebilligt. Diese Gefolgschaft steht in einer vertikalen Beziehung zum Führer und akzeptiert den entsprechenden Führer auch als Autorität. Dies spielt bei der Lösung von Konflikten und beim Schlichten von Streitigkeiten eine Rolle.

Diese hierachische Führungsstruktur, die sich mit zunehmender Bedeutung auf eine 
umfangreichere Gefolgschaft stützt, wird ergänzt durch die Achtung und die Position, die man den "Informationskontrolleuren" zubilligt. Ihr Kennzeichen ist ihre persönliche Autorität und ihr überdurchschnittliches religiös-traditionelles oder modernes Wissen. Diese Führungsqualifikation kann durchaus mit der der Dorf- und Paraführer, die oft lesen und schreiben können, verschmelzen.

Auf der "unteren" Ebene wird diese Struktur noch durch "Arbeiterführer" ergänzt. Sie zeichnen sich im allgemeinen durch gute Beziehungen zu Führern und potentiellen Arbeitgebern einerseits und andererseits durch Autorität aus, die ihnen von Arbeitern im Rahmen ihrer Vorarbeiter- und Arbeitsvermittlerfunktion zugestanden wird.

Auffälligstes Strukturmerkmal ist die hierarchisch-vertikale Struktur der verschiedenen Führungsebenen. Sie drückt sich auch aus in und ist Ausdruck sowohl des Senioritätsprinzips als auch des Patronageprinzips, das neben der sozio-politischen Dimension eine wirtschaftliche Verteilungs-, Aneignungs- und Sicherungsdimension hat. Führer zeichnen sich nicht nur durch soziales Ansehen, sondern auch durch wirtschaftliche Merkmale aus. Sie suchen sich ihre Klientel nicht nur durch eine effiziente und effektive Interessenvertretung der Klientel nach außen zu erhalten und eventuell zu vergrößern, sondern sie versuchen auch ihre Klientel durch materielle Vorteile an sich zu binden.

Die Ressourcenkontrolle allein ist nicht ausschlaggebend, sondern die Beziehungen, die ein Führer hat. Diese Beziehungen sind Ausdruck "potentieller" Ressourcenkontrolle, der das Prinzip der Optionierung, des Anmeldens und Innehabens von Ansprüchen und Rechten, zugrunde liegt.

\subsection{Faktionen und Koalitionen}

In einem Dorf gibt es niemals nur eine "Führungspyramide", sondern mindestens zwei, in der Regel mehrere. Damit tritt neben das Phänomen der Führung-Patronage noch das der Faktion und der Koalition.

Faktionen entstehen aus konkurrierenden Interessen verschiedener Führer und deren Klientel auf verschiedenen Ebenen. In der Regel geht es dabei entweder um die Ressourcenkontrolle oder um sozio-politischen Einfluß oder auch um beides. Dies drückt sich dann aus in der Größe des Gefolges, des Ansehens, das sich aus der Größe des Gefolges und der damit verbundenen Autorität ergibt, und auch in der Kontrolle über neuerlangte Ressourcen. Allgemein läßt sich das Phänomen der Faktionen, ihrer Bildung und ihrer Funktion mit dem Kampf um größere Einfluß- und Interessensphären umschreiben.

Faktionen entstehen also aus konkreten Gruppen- und Interessengegensätzen, können jedoch auch einfach aus einem persönlichen Machtstreben eines Mannes entstehen, der durch den Versuch, sich eine eigene Führungsposition aufzubauen in Konflikte mit schon etablierten Führern gerät. Er wird versuchen, seine Klientel zu vergrößern, indem er ihr Vorteile verschafft.

So gibt es also die Möglichkeit, daß eine Gruppe von Haushalten durch und mit ihrem 
Führer mit einer anderen Gruppe in Konflikt gerät, oder andererseits die Möglichkeit, daß die persönlichen Macht- und Einflußansprüche durch Klientelbildung erweitert werden. In beiden Fällen entstehen und erhalten sich Faktionen.

Faktionierung als Teilung einer Gemeinschaft in zwei oder mehr Interessengruppen kann aber auch durch Koalitionen entgegengewirkt werden. Koalitionen sind Bündnisse von zwei oder mehreren Führern zum Zweck der effektiveren Durchsetzung von für bedeutsam erkannten Interessen, die man nur gemeinsam, nicht einzeln durchsetzen kann. Diese Koalitionen richten sich in der Regel gegen einen anderen Führer oder gegen eine andere Führerkoalition.

In der Realität kann es - über einen längeren Zeitraum hin beobachtet - zu einem wechselnden Spiel von Faktionen und Koalitionen kommen. Es gibt aber immer auch langfristig schwelende alte Konflikte, die vielleicht zeitweise unbedeutend sind, aber nie völlig aufgelöst werden. Kompliziert werden kann das Interessenausgleichssystem noch durch Führerkoalitionen, die sich im Prinzip mehr oder weniger langfristig und offensichtlich gegen ihre eigene Klientel richten, zugunsten kollektiver Vorteile der Führerkoalition.

Grundsätzlich läßt sich dazu anmerken, daß bei dem reinen Kampf um Führungspositionen jeder Interessent durch Koalitionen, Schaffung von Klientel usw. versuchen wird, die der Realisierung seiner Ansprüche angemessene Strategie zu verwirklichen. Er wird abwägen, was für ihn die günstigsten Verhaltensweisen sind, und Moralität nur soweit ins Kalkül einbeziehen, als sie opportun ist.

Die politische Führung ist bis zur Paraebene hierarchisch strukturiert. Darüberhinaus sind die Führer zwar als Dorfführer bekannt, oberhalb des Paras gibt es aber keinen einzelnen allgemein anerkannten Führer mehr. Oberstes politisches Gremium ist dann traditionell nur noch der Dorfrat oder die Dorfversammlung (salis), in der verschiedene Führer gemeinsam eine Entscheidung finden. Diese Entscheidung ist theoretisch endgültig. Tatsächlich ist ihre Durchsetzbarkeit allerdings abhängig von der Position dessen, der sich der Entscheidung beugen soll.

Führung wurzelt im allgemeinen in einer lokal begründeten Basis, die verschiedene Breite und verschiedene Ebenen hat. Entsprechend den verschiedenen Ebenen werden auch Konflikte gelöst. Kleine Konflikte unter den betroffenen Haushalten selbst, Konflikte zwischen Paras oder Dörfern auf breiterer und höherer Ebene. Hier gelten die Prinzipien der segmentären Gesellschaften.

Grafik: segmentäre Struktur

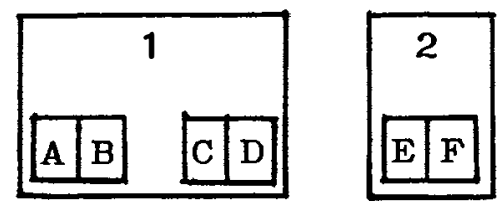


Gerät Segment A in Konflikt mit Segment B, wird dieser Konflikt unter diesen beiden Segmenten ausgetragen. Dies gilt etwa für zwei Haushalte innerhalb des gleichen Paras. Gerät $A$ jedoch in einen Konflikt mit $C$ kann es sein, daß sich B in jedem Fall mit $A$ und D mit C solidarisiert und dann der Konflikt auf dieser höheren Ebene gelöst oder ausgetragen wird. Dies gilt z. B. für verschiedene Haushalte aus verschiedenen Klans in verschiedenen Paras. Es kann dann innerhalb eines Klans oder Paras vorher noch soviel Streit gegeben haben, nach außen solidarisieren sie sich. Dieses Phänomen setzt sich dann eventuell noch auf der Ebene von Dörfern fort. Wenn z. B. Haushalt A in Dorf 1 mit Haushalt $E$ in Dorf 2 schwerwiegende Probleme hat. " Es gibt abgesehen von modernen Gerichten keine übergeordnete zentrale Autorität, die regelnd eingreifen könnte, sondern in jedem Fall müssen Ratsversammlungen einberufen werden, und die verschiedenen Führer müssen untereinander verhandeln. Das System muß sich selbst austarieren, die Interessen müssen sich gegenseitig ausbalancieren.

\section{Der sozio-politische Prozeß}

Jeder Entscheidung eines einzelnen oder einer gesellschaftlichen Gruppe gehen Meinungsbildungsprozesse voraus. Im Verlauf der Meinungsbildung wird die spätere Entscheidung schon langsam vorgezeichnet. Auch Entscheidungen entstehen in Prozessen. Trotz der Parallelität dieser beiden Prozesse sollen sie hier getrennt betrachtet werden.

\subsection{Meinungsbildungsprozesse}

Meinungsbildung setzt einen gewissen Fluß an Informationen, ein normatives Wertekonzept und eine funktionale Notwendigkeit zur Entscheidung (einen Sachzwang) voraus. Diese drei Komponenten müssen bei Existenz eines Problems zu einem Ansatz der Problemlösung führen. Vor der Lösung des Problems muß, wenn nicht durch Konsens, dann doch durch Macht, eine Handlungsanweisung entwickelt werden, wie das Problem zu lösen sei, also eine Entscheidung getroffen werden. Dies geschieht im allgemeinen in einem diffusen und langwierigen sozialen Prozeß.

Der Meinungsbildungsprozeß kann in einem gewissen Maße durch verschiedene Faktoren beeinflußt werden:

- durch die Kontrolle der in das Dorf fließenden Informationen;

- durch die soziale, religiöse oder politische Legitimität die ein Informationsträger oder -vermittler besitzt;

- durch das Ausnutzen von Abhängigkeiten wie sie z. B. durch die Kontrolle von Ressourcen entstehen;

- durch das Konkurrenzverhältnis zwischen Informations- und Ressourcenkontrolleuren, das eine gewisse Auswahl erlaubt;

11 Vgl. hierzu die Fallstudie eines dorfpolitischen Konfliktes in Herbon, D., 1984. 
- durch das Bewußtsein und die Kenntnis dieser Einflüsse und der daraus entstehenden eigenen Machtposition.

In diesem Spannungsfeld von Faktoren, die hier nicht vollständig und umfassend dargestellt werden können, spielt sich ein Meinungsbildungsprozeß ab. Hinzu kommt ein allgemeines Meinungsklima oder auch soziales Klima, in dem der Prozeß der Meinungsbildung abläuft. Dieses "Meinungsklima" besteht in gewisser Weise aus den Erfahrungen, die man mit Meinungen und Meinungsbildungsprozessen in der Vergangenheit gemacht hat. Ständiger "Klatsch und Tratsch" im Dorf läßt auch erkennen, wie das allgemeine Stimmungsbild aussieht. Diese Stimmung wirkt auf die neuen akuten Meinungsbildungsprozesse ein. Der Meinungsbildungsprozeß und die Lenkung und Kontrolle von Informationen ereignet sich vor einem breiten Spektrum verschiedener Interessen.

Jede Entscheidung geht also mit einer sorgfältigen Úberprüfung von eigenen Interessen, den möglichen Vor- und Nachteilen, einer Bilanzierung der dörflichen politischen Machtverhältnisse usw. einher. Führer versuchen für sie negative Entscheidungen zu verhindern. Ungewünschte Entscheidungen sind jedoch nicht wahrscheinlich, wenn man allen Widerstand vorher gekannt und eingeplant hat.

Meinungsbildungsprozesse stellen ein sehr komplexes Netzwerk mit Rückkopplungen dar, in dem sich im Laufe der Zeit eine Meinung und eine Lösung "herausschält". Hierbei wirken viele soziale, wirtschaftliche, religiöse und normative Faktoren zusammen und formen bei jedem Problem die Entscheidungs- und Meinungsbildungsstruktur neu.

\subsection{Entscheidungsstrukturen}

Der Begriff "Entscheidungsstruktur" ist komplex und diffus zugleich. Jeder menschlichen Handlung geht eine Entscheidung - eben so oder anders zu handeln - voraus. Die Entscheidung kann bewußt, aber auch unbewußt gefällt werden. Entscheidungen werden zudem nicht nur von Personen selbst, sondern eventuell auch von einer Gruppe nach Diskussion in einem Meinungsbildungsprozeß gefällt. Im täglichen Dorfleberı müssen eine Fülle von Entscheidungen gefällt werden.

Jeder Haushaltsvorstand, (Entscheidungsträger) entscheidet in einer ihm optimal für seine Familie erscheinenden Weise. Er entscheidet nach verschiedenen wirtschaftlichen, sozialen und kognitiven Kriterien, die ihm wichtig sind. In jedem dieser Bereiche und im Verhältnis jedes dieser Bereiche zu anderen Bereichen wird er seine Zielstruktur zu optimieren bemüht sein. Jeder Haushalt wird zunächst einmal entsprechend seiner Zielstruktur und der Struktur seiner Ausstattung seine eigenen Bedürfnisse zu befriedigen suchen bzw. die seines Haushaltes. Soziale Organisation, Zusammenarbeit, aber auch Konflikt sind aus diesem Bedürfnis abgeleitet, sie sind sekundär und den individuellen Zielsetzungen untergeordnet. Individuelle Interessen sind also auch bei sozialen Gruppenentscheidungen ausschlaggebend. Soziale Zusammenschlüsse, in welcher Form 
auch immer, werden nur so lange unterstützt, wie sie dem einzelnen unter dem Strich mehr Nutzen bringen, als wenn er auf sich gestellt wäre.

$\mathrm{Zu}$ der Tatsache, daß der einzelne Mitglied sozialer Gruppen ist, kommt aber noch eine weitere Erwägung hinzu, nämlich die des Gruppeninteresses. Es kann sich für den einzelnen Haushalt durchaus als sinnvoll erweisen, ein definiertes Gruppenziel zu verfolgen oder allgemeine Gruppeninteressen zu vertreten, weil er weiß, daß dies auch ihm kurz- oder langfristig nützt. Eine typische Gruppe, deren Interessen zunächst langfristig als die eigenen angesehen werden, ist die Verwandtschaftsgruppe. Umgekehrt muß auch eine von dieser Gruppe anerkannte Führungspersönlichkeit nicht nur ihr eigenes Interesse im Auge haben und zu realisieren versuchen, sondern auch das der Gruppenmitglieder im Blick haben und gleichzeitig den Gruppenmitgliedern diesen Eindruck vermitteln. Die Zielorientierung des einzelnen ist damit auch immer gruppenbezogen, was der primären eigenen Zielrealisierung keinen Abbruch tun muß.

Festzuhalten ist, daß Entscheidungen nie altruistisch sind, sondern klaren Interessen oder aber Zwängen folgen. Dieser im Grunde hierarchischen Interessendurchsetzungsund Entscheidungsstruktur liegen bis zu einem gewissen Grade die Prinzipien des Klientelismus, aber auch der Kooperation und besonders auch der Segmentation zugrunde. Entscheidungen werden auf der niedrigst möglichen Ebene, d. h. von der kleinstmöglichen sozialen Einheit im eigenen Interesse getroffen.

\subsection{Schilderung eines idealtypischen sozio-politischen Entscheidungs- und Meinungs- bildungsprozesses}

Im Untersuchungsdorf gab es ständig Neuigkeiten und Probleme, die besprochen und diskutiert wurden. Nicht alle betrafen das gesamte Dorf. Müssen die Menschen in irgendeiner Form auf das Problem reagieren, d. h. sich eine Meinung bilden und dann zu einer Entscheidung kommen, lassen sich idealtypisch die folgenden Abläufe feststellen. Durch Gespräche auf einer Veranda, unter einem großen Mangobaum, durch Klatsch der Frauen usw. wird das Problem hin und her gewendet. Meinungen werden nicht definitiv geäußert, sondern jeder hält sich zurück. Statusniedere Personen vermeiden es, so lange sie nicht selbst betroffen sind, einen Standpunkt zu beziehen - der brächte ihnen ja auch nicht viel ein. Um ihre Meinung befragt, antworten sie ausweichend, da müßten sich erst "zehn Männer zusammensetzen". Führer halten bei den langfristigen - im Gegensatz zu den akuten - Problemfällen nicht so sehr mit ihrer Meinung zurück.

Die Häufigkeit der Konsultationen der Führer untereinander nimmt zu, ein Abschätzen und Verrechnen der Gefolgezahlen findet statt, und das Problem wird hin und her gewendet. Die Meinungsbildung findet also, lange bevor die Entscheidung deutlich wird, "hinter den Kulissen" statt. Es gibt kein öffentliches Forum oder Medium, in dem in irgendeiner formalisierten Form diskutiert wird. Das wäre ja auch nicht so günstig, denn bei öffentlichen Äußerungen wäre man festgelegt, angreifbar und verlöre an Ansehen, wenn man von seiner geäußerten Meinung wieder abrücken müßte.

Gleichzeitig findet eine Definition der eigenen Interessen und ein Abwägen der Interes- 
sen anderer statt. Dabei wird unterschieden zwischen Interessen des eigenen Haushaltes und der eigenen Gefolgschaft und denen der Konkurrenten.

Entscheidungen werden von Führern nicht autokratisch gefällt. Die Betroffenen haben immer auch etwas dazu zu sagen. In der Grundtendenz wird nicht dauerhaft gegen den Willen der Gefolgschaft entschieden, sondern in ständiger Rückkopplung mit der Gefolgschaft bzw. mit Koalitionspartnern. Es wird nach einem längeren Abwägen des Für und Wider, durch ein Hin- und Herwenden der Argumente eine Art Gruppenmeinung artikuliert oder indirekt zum Ausdruck gebracht. Die Kunst eines Führers liegt nun darin, aufmerksam zuzuhören, seine Meinung nicht sofort kundzutun, sich bedeckt zu halten, und wenn er erkennen kann, wohin sich die Waage der Meinungen neigt, als erster das Diskussionsergebnis zu formulieren und das Diskussionsergebnis als seinen originären Vorschlag zur Diskussion einzubringen. Er bringt damit eine latent schon existierende, manchmal aber nicht bewußte oder zumindest nicht formulierte "öffentliche" Meinung zum Ausdruck. Die Gefolgschaft stimmt ihr dann natürlich zu, weil es im Grunde ihre eigene Meinung ist. So zeigt er zum einen seine "Weisheit" und zum anderen seine "Fürsorglichkeit", denn er erkennt ja die Interessen der Gefolgschaft. Dies festigt seine Führungsposition.

Führer ergreifen bei irgendwelchen Treffen immer erst relativ spät das Wort, sitzen meist sogar am "Rande des Zentrums" und lassen erst die unterschiedlichen Meinungen bis zur Ratlosigkeit aufeinander prallen. Je höher ihr Status ist, desto später äußern sie sich.

Die Entscheidungen spitzen sich also im Laufe des Meinungsbildungsprozesses "trichterförmig" zu, und an der Spitze bzw. am Ende des Prozesses steht die Aussage des Führers, die dann als Entscheidung respektiert wird und einen autoritären Eindruck erweckt. Dieser Eindruck stimmt aber so nicht. Einerseits kann der Führer es sich nicht leisten, seine Gefolgschaft in eine Richtung zu pressen, die ihr ganz und gar nicht paßt. Beeinflussen kann er sie aber wohl. Das kann er, wenn er frühzeitig an der richtigen Stelle und gegenüber der richtigen Person doch einmal kund tut, was er will, sich dann jedoch zurückhält und die Wirkungen abwartet. Andererseits wird, wenn es sich um einen anerkannten Führer handelt, seine Entscheidung - zumindest für den gegebenen Fall - als endgültig angesehen. Der Anschein autokratischer Entscheidungen durch einige wenige Führer muß daher relativiert werden, denn dörfliche Konsensbildung und informale Klärungsprozesse gehören ebenso zum Erscheinungsbild, wie hierarchische Patron-Klient- und Führungsstrukturen.

Macht als Fähigkeit, eigene Entscheidungen durchzusetzen, ist dabei immer relativ zur Position des Gegenspielers zu sehen. Je größer der Unterschied in der Position, die jeweils bestimmt ist durch Kontrolle von Ressourcen, Einkommen, Wissen und a priori Macht, desto unbalancierter oder asymmetrischer wird die Entscheidung ausfallen. 


\section{Zusammenf assende Schlußbetrachtung}

Im Untersuchungsdorf war eine starke Tendenz zur Entscheidungsautonomie der einzelnen Gruppen nach den oben dargestellten Prinzipien festzustellen. Dieses segmentäre Prinzip ist, soweit es den traditionellen Ablauf des Dorflebens betrifft, nicht weiter problematisch. Probleme tauchen erst dann auf, wenn die normalerweise agierende größte Einheit sich im Rahmen der modernen Anforderung als zu klein erweist, wie z. B. bei der Anlage und Wartung von Bewässerungssystemen und der Organisation von Genossenschaften usw.

Allgemein läßt sich sagen, daß im Bereich der Agrarproduktion jeder Haushalt, soweit er die Ressourcen, die er braucht, kontrolliert, seine eigenen Entscheidungen trifft, die seine persönlichen Interessen betreffen. Kooperative Strukturen, die gemeinschaftliche Entscheidungsfindung erfordern, sind unbedeutend. Normalerweise reichen dazu die dyadischen Entscheidungen beim Austausch zwischen den Haushalten aus. Komplexere Entscheidungsstrukturen sind jedoch bei sozialen Verteilungsmechanismen von Einkommen im Dorf oder von Ressourcen, die von außen ins Dorf fließen, nötig. Zur Beeinflussung und Förderung von Entwicklung und Partizipation ist eine detaillierte Kenntnis sozio-politischer Strukturen und Prozesse in Dörfern entscheidend. 
The Malvinas Conflict: Some Elements for an Analysis of the Argentinian Military Regime's Decision-making Process (1976-1983)

\section{By Carlos J. Moneta}

The purpose of this paper is to present some reflections on the decision-making process of the Argentinian military government in relation to the South Atlantic conflict, which may serve as a contribution to a more exhaustive study in the future. It also involves a recognition of the impact of the Malvinas conflict on the national and international scene.

The paper analyses the characteristics of the political regime in power, its goals, values and the means applied to reshape Argentine society; the concentration of the decisionmaking process in a very limited number of actors, most of whom belong to only one type of organization, namely the armed forces; the exploitation of a national cause - the recovery of the Malvinas - to obtain support and international political legitimacy for the regime which was at that time facing massive serious social and political unrest. It also examines the particular ideologies of the leadership and the problems of competition between them and between their respective organizations.

Special attention is given to the Argentine military leaders' distorted perception of the international system, the East-West confrontation and the ways by which Argentina may improve its strategic stature achieving at the same time an historical goal: To recover part of the national territory under British occupation since the 19th century.

Finally, the paper briefly explores the long-term political impact that the Malvinas war could have upon Argentinian society in a context where it is attempting to restore democracy.

\section{The Political Structure of a Bangladesh Village}

\section{By Dietmar Herbon}

The integration of the rural population into the processes of development, or rather of solving multiple problems, in Bangladesh requires adequate social and political forms of participation.

Knowledge of present conditions and structures is a prerequisite for corresponding concepts and strategies. Based on an empirical investigation, political structures of a Bangladesh village are described and analysed.

Firstly, single positions, criteria and levels of the leadership structure are discussed and the corresponding types of leaders presented.

Secondly, the leader and follower structure of the whole system is shown, including the fact that in the village more than just one "pyramid of leader and follower" exists, and that they form variable coalitions and factions competing with each other. 
Finally, an attempt is made to show the formation processes of public opinion and decisions.

Characteristics of the political structure in the Bangladesh village thus are:

- a strong tendency towards autonomy of decisions of households and groups and, thus,

- a segmentary structure which leads to

- a lacking or limited ability to cope with more complex tasks and challenges.

\section{Swaziland's Proposed Land Deal with South Africa - The Case of Ingwavuma and Kang- wane}

\section{By Wolfgang Senftleben}

In mid-1982 a proposal was made public to incorporate a substantial land area of South Africa's tribal homelands of Kangwane and KwaZulu into the Kingdom of Swaziland. If such a transaction should be completed, it would give hitherto land-locked Swaziland access to the sea with a potential port at Kosi Bay, and would incorporate all areas of ethnic Swazi population into the kingdom. In return for South Africa the land transfer would mean a tacit approval of her apartheid policy, besides the advantage of creating a buffer zone against Marxist-orientated Mozambique. However, vehement opposition against the land transfer has been registered internally from the native population involved as well as from the international community, particularly from the O.A.U. Recent legal setbacks through court intervention in South Africa, the current power struggle in Swaziland after the death of King Sobhuza II, as well as South Africa's impending change of the parliamentary system and her recent diplomatic and political initiatives towards her neighbours, have shelved the land transfer issue for the time being. However, the land deal, if it succeeds, would constitute an exchange unprecedented in world history. 